\title{
Statistical Model Checking of Complex Robotic Systems
}

\author{
Mohammed Foughali $^{1}$, Félix Ingrand ${ }^{1}$, and Cristina Seceleanu ${ }^{2}$ \\ 1 LAAS-CNRS, Université de Toulouse, CNRS, Toulouse, France \\ mfoughal@laas.fr, felix@laas.fr \\ 2 Mälardalen University, Västerås, Sweden \\ cristina. seceleanu@mdh. se
}

\begin{abstract}
Failure of robotic software may cause catastrophic damages. In order to establish a higher level of trust in robotic systems, formal methods are often proposed. However, their applicability to the functional layer of robots remains limited because of the informal nature of specifications, their complexity and size. In this paper, we formalize the robotic framework $\mathrm{G}^{\mathrm{en}}{ }_{\mathrm{oM}} \mathrm{M}$ and automatically translate its components to UPPAAL-SMC, a real-time statistical model checker. We apply our approach to verify properties of interest on a real-world autonomous drone navigation that does not scale with regular UPPAAL.
\end{abstract}

\section{Introduction}

Although robotic software is tested, both in the field and using simulators, its lack of safety hinders the deployment of robots in costly and human-interaction missions (e.g. home assistants, deep space). As an example, the NASA Remote Agent Experiment had to be stopped due to a deadlock, never detected during the one-year testing phase [25]. Other examples include the autonomous vehicle Alice [19] and the museum guide RoboX9 [32]. Such failures are mainly due to the nature of classical, scenariobased testing, unable to provide guarantees on important properties. Formal methods are a promising alternative, but their use in robotics is still marginal, and varies according to the software layers [33]. Indeed, at the decisional layer, in charge of high-level decision making functions (e.g. planning [17]), models are often formal with complete semantics, which facilitates their formal modeling and verification [7, 12]. In contrast, functional layer components, in charge of low-level actions involving sensors and actuators (e.g. localization and navigation), are developed within non formal frameworks (e.g. ROS [26]), which makes their formalization particularly challenging and costly. Furthermore, the formal modeling is non reusable (it needs to be redone whenever a component evolves) and models are not guaranteed to scale. Consequently, many previous works either focus on simple case studies (usually not deployed on real robots), resort to non realistic abstractions (e.g. ignoring timing constraints), or propose no alternatives to deal with scalability issues (Sect. 7).

We propose in this paper the use of formal methods to verify the functional layer of robotic systems. We focus on verification by means of model checking, and use statistical model checking [22] to tackle scalability issues. A particular interest is given to real-time properties, e.g. schedulability and bounded response, crucial in robotics (examples in Sect. 6). To tackle the abovementioned problems, we (1) formalize (Sect. 3) the robotic framework GenoM3 (Sect. 2), (2) develop automatic, sound transformation 
from any $\mathrm{G}^{\mathrm{e}}{ }_{\mathrm{OM}} \mathrm{3}$ specification into UPPAAL and UPPAAL-SMC models (Sect. 4, 5) and (3) verify crucial real-time properties, while avoiding non-realistic abstractions (e.g. all timing constraints are considered), on a real drone application (Sect. 6). We conclude with related work (Sect. 7) and lessons learned (Sect. 8).

\section{Preliminaries}

\section{$2.1 \mathrm{G}^{\mathrm{en}} \mathrm{OM} 3$}

$\mathrm{G}^{\mathrm{en}}{ }_{\mathrm{o}} \mathrm{M} 3$ [23] is a tool to specify and implement robotic functional components. Each component, in charge of a functionality, ranging from sensor control (e.g. laser) to more integrated computations (e.g. navigation), is organized as shown in Fig. 1a. For space and readability, we omit in this paper control services and interruption of activities, but the interested reader may refer to [11] for details.

A component implements the core algorithms of its functionality within activities, which it executes following requests from external clients. Thus, the component has a (i) control Task to process the clients requests and report to them accordingly and (ii) one or more execution task(s) to execute activities. These tasks share parameters and computed values of the component through the Internal Data Structure (IDS). Finally, a component provides ports to share data with other components.

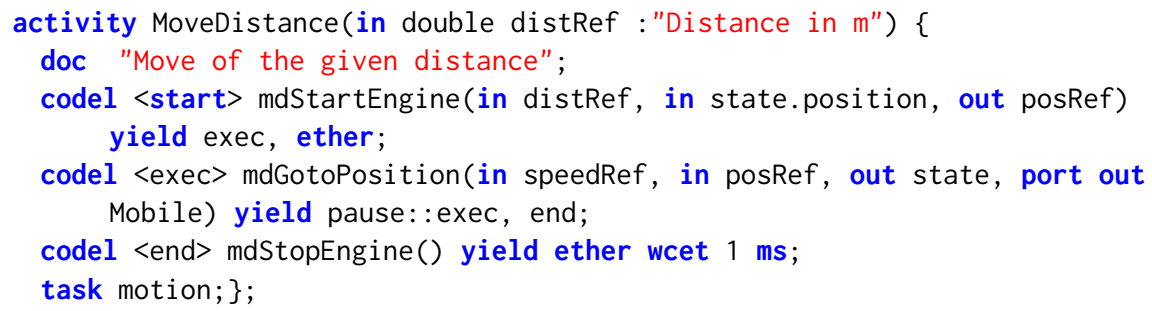

Listing 1: Activity MoveDistance

2.1.1 Behavior We briefly explain how a component behaves. We use the support example of activity MoveDistance that belongs to the component DEMO, developed for illustration purposes (listing. 1).

Activities: activities are finite-state machines FSM, each state called a codel. An activity is executed by the execution task it specifies (line 6 specifies that activity MoveDistance is executed by the motion task).

FSM: define the activity behavior through codels and transitions. A codel is a state at which a chunk of $\mathrm{C}$ or $\mathrm{C}++$ code is executed. It specifies its arguments (e.g. exec uses the IDS fields speedRef, posRef and state and the port Mobile, line 4) and the possible transitions subsequent to its execution (e.g. start returns exec or ether, line 3). Taking a pause transition pauses the execution of the activity until the next cycle (see below) of its execution task (e.g. taking transition pause: : exec, line 4, pauses the activity at codel exec from which it will be resumed at the next cycle of task motion). A codel may (optionally) specify a WCET (worst case execution time) on a given platform (e.g. end has a WCET of $1 \mathrm{~ms}$, line 5). An FSM has always the codels start (entry point) and ether (end point with no code attached). When the latter is reached, the activity is terminated and reported to the client. 


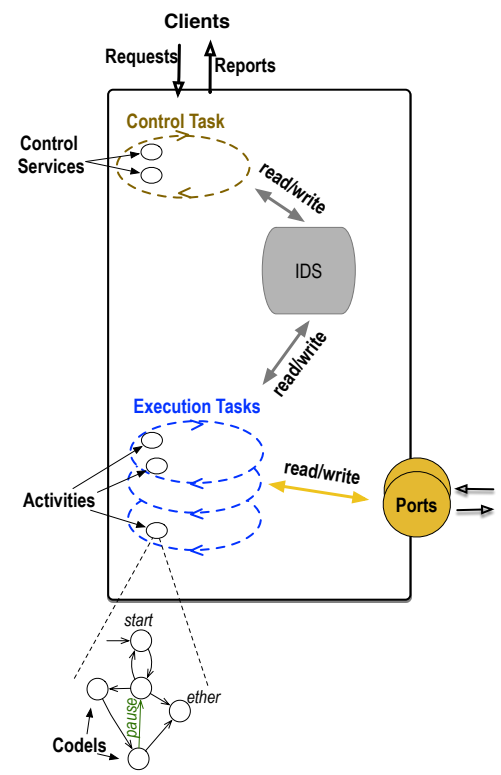

(a) A generic $\mathrm{G}^{\mathrm{e}}{ }_{\mathrm{o}} \mathrm{M} 3$ component

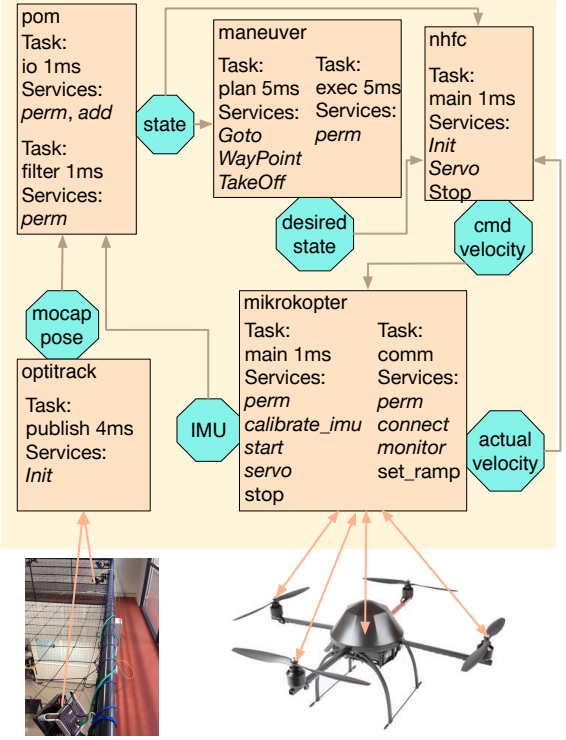

(b) The quadcopter case study

Fig. 1: Generic GenoM3 component \& case study.

Control task: manages requests and reports (from/to clients). When a request for an activity is received, the control task validates it and activates such activity (which informs the execution task in charge to execute it). Upon completion of any activity, the control task sends a report to the corresponding client.

Execution tasks: periodic or aperiodic. With each cycle (triggered by period or event), an execution task runs, sequentially, all the activities it is in charge of, previously activated by the control task. The execution of an activity ends when it is paused or terminated. In the former case, the activity is resumed at the next cycle.

IDS \& concurrency: Tasks are run as parallel threads, with fine-grain concurrent access to the IDS: only the required field(s) by a codel (in its activity, run in a task) are locked when it executes and simultaneous readings are allowed.

2.1.2 Templates $\mathrm{G}^{\mathrm{e}}{ }_{\mathrm{O}} \mathrm{M} 3$ features an automatic generation mechanism based on templates. A template may access all the component information (e.g. tasks periods, activities and their codels) and generate text files with no restrictions (examples in Sect. 5.2). There are templates that, for instance, generate component implementations for PocoLibs [1] and ROS-Comm [26] middleware. These implementation templates also collect codels execution time, which are reported (average and WCET) upon completion, and the number of occurrences of transitions in all activities (Sect. 5.2).

2.1.3 Case study In this paper, we consider the quadcopter in Fig. 1b. In Sect. 6, we explain how we use the components for a navigation mission. For technical details on each component (out of the scope of this paper), we refer the interested reader to [8]. 


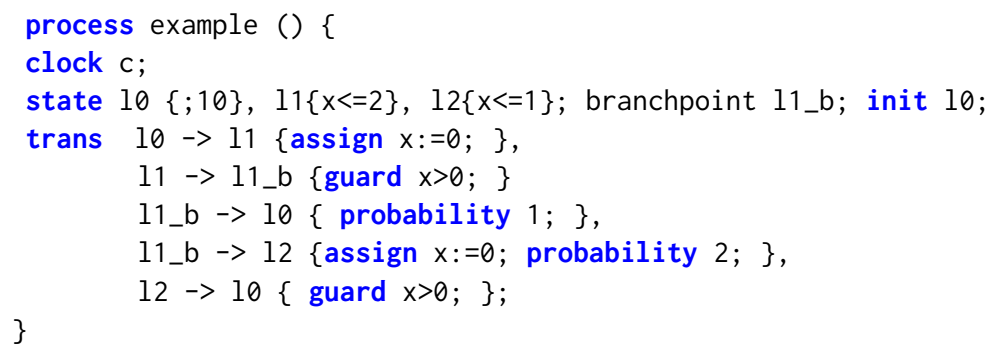

Listing 2: STA example in .xta format

\subsection{UPPAAL}

UPPAAL [2] is a real-time model checker. Models are based on timed automata (TA) and supported properties are mainly safety, liveness and bounded response.

Timed Automata: A TA [16] is a tuple $\left\langle L, l_{0}, X, \Sigma, E, I\right\rangle$ where $L$ is a finite set of locations, $l_{0} \in L$ is the initial location, $X$ is a finite set of clocks, $\Sigma$ is a finite set of actions including synchronization and internal actions, $E$ is a finite set of edges of the form $\left(l, g, a, \varphi, l^{\prime}\right)$, with $l, l^{\prime} \in L, g$ a predicate on $\mathbb{R}^{X}, a \in \Sigma$, and $\varphi$ a binary relation on $\mathbb{R}^{X}$, and $I$ assigns an invariant predicate $I(l)$ to any location $l$.

Extending TA: In a TA, urgencies are expressed locally through invariants. For global urgencies, e.g. involving different TA, UTA [4] are introduced. In a UTA, when an eager edge (denoted $\zeta$ ) is enabled, time cannot progress and the edge must be taken (or disabled by taking another edge) immediately. TA can also be extended with data variables. We refer to UTA extended with data as DUTA. Fig. 2 shows a DUTA example with two locations, $l 0$ (initial, denoted with an inner circle), and $l 1$, and one ₹edge. Guards are in green, invariants in purple and operations in blue. ex (resp. $O$ ) is a Boolean expression (resp. some operations) over some variables. In this example, if the guard remains false for more than 3 time units, the DUTA timelocks.

UPPAAL supports a subclass of DUTA that allows (i) urgent channels (over which only time-constraint-free edges may synchronize), but not eager edges (example in Sect. 5) and (ii) Boolean and integer data types and functions without pointers.

\subsection{UPPAAL-SMC}

UPPAAL-SMC is an extension of UPPAAL based on stochastic timed automata STA.

Stochastic Timed Automata: An STA is a tuple $\langle T A, \mu, \gamma\rangle$ where

$T A=\left\langle L, l_{0}, X, \Sigma, E, I\right\rangle$ is a timed automaton (Sect. 2.2), $\mu$ is the set of density delay functions $\mu_{s} \in L \times \mathbb{R}^{X}$, which can be either uniform or exponential distribution, and $\gamma$ is the set of probability functions $\gamma_{s}$ over $\Sigma$ in $T A$.

In brief, STA extend TA with (i) density functions (on locations) and (ii) probabilities (on edges). Since we target STA as supported by UPPAAL-SMC, we show an STA example in the .xta format (listing 2). If the location has an associated invariant (e.g. $l 1$, line 3 ), the density function is a uniform distribution (exponential distribution with a user-supplied rate otherwise, e.g. 10 on $l 0$, line 3). Probabilities, uniform by default, can be added using (i) a branchpoint (lines 5 to 7) and (ii) the keyword "probability" followed by the number of occurrences, used to compute probabilities (the probability to take the edge from $l 1$ to $l 0$ (resp. to l2) is $1 / 3$ (resp. 2/3)). 
Verification in UPPAAL-SMC: In this paper, we are interested in probability evaluation, that is estimating the probability $\operatorname{Pr}[<=b]\left(O p_{x \leq d} \phi\right)$ where $b$ is a time bound on runs, $O p$ is either $\diamond$ or $\square$ and $\phi$ lies within the Weighted Metric Temporal Logic $W M T L_{\leq}[5]$ grammar (atomic propositions endowed with $U$, the until operator and $O$, the next operator).

\section{Formalizing $\mathrm{G}^{\mathrm{en}}{ }_{\mathrm{O}} \mathrm{M} 3$}

We semanticize Gen ${ }_{0} \mathrm{M} 3$ components using timed transition systems TTS (Sect. 3.1). For readability and space, control task and aperiodic behaviors are excluded. This version preserves important mechanisms, e.g. concurrency, and the more complex version can be found in [11]. Since the control task is excluded, we will often refer to an execution task as simply task.

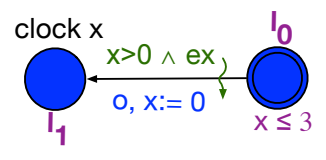

Fig. 2: A generic DUTA example

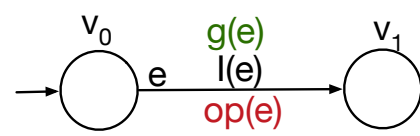

Fig. 3: A generic TTD example

\subsection{Timed transition systems}

We propose a variation of TTS in [15] where (i) a dense-time model (time intervals have durations in $\mathbb{R}_{\geq 0}$ with bounds in $\mathbb{Q}_{\geq 0} \cup \infty$ ) is considered instead of a discrete one and (ii) more general time intervals (left- and right-open) are accepted. TTS are suitable to semanticize $\mathrm{Gen}_{\mathrm{o}} \mathrm{M} 3$. For instance, they are convenient to formalize the global urgency constraints (e.g. a codel executes as soon as it has the required (shared) resources, Sect. 2.1.1), as opposed to clock-based transition systems such as TA where urgencies are expressed only locally (see examples in [8]). Semantics in TTS also allowed automatic mapping to Fiacre in [10].

Let $\mathbb{I}$ be the set of well-formed (time) intervals. An element $i$ of $\mathbb{I}$ can have the form: (f1) $[a, b]$ (f2) $] a, b]$ (f3) $\left[a, b[\right.$ or (f4) $] a, b\left[\right.$, where $a \in \mathbb{Q}_{\geq 0}, b \in \mathbb{Q}_{\geq 0} \cup \infty$, and with $a \leqslant b$ for $\mathrm{f} 1$ ( $a<b$ otherwise). Interval $i$ is thus the set of reals $x \in \mathbb{R}_{\geq 0}$ such that $a \leq x \leq b$ (f1), $a<x \leq b$ (f2), $a \leq x<b$ (f3), $a<x<b$ (f4). In any form, we say that $\downarrow i=a$ (resp. $\uparrow i=b$ ) is the lower (resp. upper) bound of $i$.

A TTS is a tuple $\left\langle U, S, s_{0}, \tau, I\right\rangle$ where:

- $U$ is a finite set variables,

- $S$ is a set of states. Each state of $S$ is an interpretation of variables in $U$,

- $s_{0}$ is the initial state $\left(s_{0} \in S\right)$ that maps each variable in $U$ to its initial value,

$-\tau$ is a set of transitions. Each transition $t \in \tau$ defines for every state $s \in S$ a (possibly empty) set of successors $t(s) \subseteq S$,

- $I: \tau \mapsto \mathbb{I}$ maps each transition $t \in \tau$ to a static (time) interval $I(t) \in \mathbb{I}$.

The semantic "meaning" of time intervals depends on the enabledness of transitions: if transition $t$ is enabled at $s$ ( $s$ is the current state of the TTS and $t(s) \neq \varnothing$ ) since date $\Delta$ then we can take $t$ starting at date $d$ s.t. $\Delta+\downarrow I(t)<d$ if $I(t)$ is of form (f2) or (f4) ( $\Delta+\downarrow I(t) \leq d$ otherwise) and must take it no later than date $d^{\prime}<\Delta+\uparrow I(t)$ if $I(t)$ is of form (f3) or (f4) ( $d^{\prime} \leq \Delta+\uparrow I(t)$ otherwise), unless it is disabled in between 
by taking another transition. If $t$ is disabled, then $I(t)$ has no semantic effect (detailed semantics in [11]).

3.1.1 TTDs A timed transition diagram TTD (inspired from [15]) is a finite directed graph with a set of vertices $V$ and a set of edges $E$. The unique initial vertex is $v_{0} \in V$. Each edge $e \in E$ is labeled with: an interval $I(e)$ (omitted if equal to $[0, \infty[$ ); a guard $g_{e}$ (omitted if tautology); and an atomic sequence of operations $o p_{e}$ (omitted if has no side effects). An edge $e$ connecting vertex $v$ to vertex $v^{\prime}$ is denoted, interchangeably, $e \in E$ or $v \stackrel{e}{\rightarrow} v^{\prime} \in E$. Fig. 3 shows a simple generic TTD with two vertices, $v_{0}$ (initial, denoted with an incoming edge without source) and $v_{1}$, and one edge $e$.

3.1.2 Composition of TTDs The parallel composition of $n$ TTDs, $P_{1}, \ldots, P_{n}$, over a set of shared variables, $U_{s}$, results in a TTS $\{\Theta\}\left[\|_{i \in 1 . . n} P_{i}\right]$, where $\Theta$ gives the initial valuations of each variable in $U_{s}$ and each component $P_{i}$ accesses $U_{s}$ and a set of local variables $U_{i}$. For detailed semantics of such TTS, we refer the interested reader to [11].

For simplicity, we stop referring to the names of edges in TTDs: $v \stackrel{e}{\rightarrow} v^{\prime}$ (Sect. 3.1.1) will be referred to, from now on, as simply $v \rightarrow v^{\prime}$, or $v \rightarrow$ (resp. $\rightarrow v^{\prime}$ ) when the identity of $v^{\prime}$ (resp. $v$ ) is irrelevant. This is because in our Gen ${ }_{\circ}$ M3 semantics (Sect. 3.3), edges are uniquely defined through their source and target vertices.

\subsection{Syntax and syntactical restrictions of a $\mathrm{G}^{\mathrm{en}}{ }_{\mathrm{O}} \mathrm{M} 3$ component}

3.2.1 Activity An activity $A$ is a tuple $\left\langle C_{A}, W_{A}, T_{A}, T_{A}^{P}\right\rangle$ where:

- $C_{A}$ is a set of codels with at least two codels (for starting and termination, Sect. 2.1.1): $\left\{\operatorname{start}_{A}\right.$, ether $\left._{A}\right\} \subseteq C_{A}$,

- $W_{A}: C_{A} \backslash\left\{\right.$ ether $\left._{A}\right\} \mapsto \mathbb{Q}_{>0}$ associates to every codel its WCET (Sect. 2). The codel ether $_{A}$ (reserved for termination) is excluded (no code attached to it, Sect. 2.1.1),

- $T_{A}$ is a set of transitions of the form $c \rightarrow c^{\prime}$ (each transition is uniquely defined through its source codel $c$ and target codel $c^{\prime}$ ). We denote this relation by simply $c \rightarrow$ (or $\rightarrow c^{\prime}$ ) when the identity of codel $c$ (or $c^{\prime}$ ) is unimportant,

- $T_{A}^{P} \subseteq T_{A}$ is the set of pause transitions.

3.2.2 Task A task $T$ is a triple $\langle\operatorname{Per}, \mathcal{A}, V\rangle$ where:

- Per $\in \mathbb{Q}_{>0}$ is the period,

- $\mathcal{A}$ is the non-empty set of activities $T$ is in charge of,

$-V$ is a set of variables.

3.2.3 Component A component Comp is a triple $\langle E, V, \mu\rangle$ where:

- $E$ is a set of tasks,

- $V$ is a set of variables,

- $\mu: C \mapsto \mathcal{P}(C)$ is the conflict function, where $C$ is the union of all codels in all activities of all tasks in $E$ and $\mathcal{P}(C)$ its powerset. $\mu(c)$ is the set of codels that are in conflict (cannot execute simultaneously) with $c$. If $\mu(c)=\varnothing$ then $c$ is thread safe (thread unsafe otherwise).

3.2.4 Well-formed components Well-formed components are defined by the following syntactic restrictions. For any activity $A$, we require that (i) each codel in $C_{A} \backslash\{$ ether $A\}$ has at least one successor in the relation defined by $T_{A}$, (ii) $T_{A}$ must not include any transition whose source codel is ether $_{A}$ (reserved for termination), and (iii) ether $_{A}$ cannot be the target of a pause transition because the latter is for pausing 
while the former is for termination. These requirements can be expressed succinctly as follows:

$$
\begin{aligned}
& \forall c \in C_{A} \backslash\left\{\text { ether }_{A}\right\} \exists c^{\prime} \in C_{A}:\left(c \rightarrow c^{\prime} \in T_{A}\right) \\
& \forall c, c^{\prime} \in C_{A}:\left(c \rightarrow c^{\prime} \in T_{A}\right) \Rightarrow\left(c \neq \text { ether }_{A}\right) \\
& \forall c, c^{\prime} \in C_{A}:\left(c \rightarrow c^{\prime} \in T_{A}^{P}\right) \Rightarrow\left(c^{\prime} \neq \text { ether } A\right)
\end{aligned}
$$

Finally, ether codels are thread safe. Also, there is no conflict within the same task: any two activities $A$ and $B$ in the same task are executed sequentially "by construction" (one task $=$ one thread). Therefore, we require that $\mu(c) \cap C_{B}=\mu\left(c^{\prime}\right) \cap C_{A}=\varnothing$ for all $c$ in $C_{A}$ and $c^{\prime}$ in $C_{B}$.

\subsection{Operational semantics of a $\mathrm{G}^{\mathrm{en}}{ }_{\mathrm{o}} \mathrm{M} 3$ component}

Before we go further, we need to distinguish between what the programmer specifies (reflected at the syntactical level, e.g. in transitions $T_{A}$, Sect. 3.2.1), and what is enforced to produce the expected behavior (e.g. starting and mutual exclusion edges, Definition 3). We present operational semantics "top-down", from component to activities.

3.3.1 Component semantics A component Comp semantics is given by the TTS Comp $=\{\Theta\}\left[\|_{i \in 1 . . n} T_{i}\right]$ where $n=|E|$ is the number of tasks in $E$ (Sect. 3.2.3) and $T_{i}$ are tasks. For each codel $c \in C$ s.t. $\mu(c) \neq \varnothing$ (Sect. 3.2.3), there is a Boolean $r_{-} c$ in the set of shared variables $U_{s}\left(V\right.$ in Sect. 3.2.3), initially false $\left(\Theta\left(r_{-} c\right)=\right.$ False for all $r_{-} c \in U_{s}$ ). These variables help semanticize concurrency (Definition 3).

3.3.2 Task semantics. The semantics of a task is given by the TTS $T=\{\Theta\}\left[\operatorname{Tim}\|M\|\left(\|_{A \in \mathcal{A}} A\right)\right]$ where Tim is the timer (Definition 1), $M$ is the task manager (Definition 2), and $\|_{A \in \mathcal{A}} A$ is the composition of all activities $A$ (Definition 3) in $\mathcal{A}$ (Sect. 3.2.2). The set of shared variables $U_{s}(V$ in Sect. 3.2.2) contains: $N$, the set of "names" of activities to execute, sig, the period signal, and $\Pi$, the control passing variable. $\Pi$ ranges over TTDs "names" (by abuse of notation, $M$ is the name of the manager TTD and the name of activity $A$ is $A$ ), $N$ has the same type as $\Pi$ excluding $M$, and sig is a Boolean. The initial values are $\Theta(N)=\varnothing, \Theta($ sig $)=$ False, and $\Theta(\Pi)=M$ (the manager has the control when the system starts).

Definition 1 Timer semantics. The timer semantics is given in Fig. 4.

Changing the value of sig to true corresponds to transmitting a signal asynchronously to the manager (Definition 2). The time interval $[$ Per, Per $]$ ensures that this signal is transmitted at exactly each period (each $P e r$ time units).

Definition 2 Manager semantics. The manager semantics is given in Fig. 5.

Vertex wait denotes waiting for the next period and manage is to execute activities, if any. The operation $\Pi:=\operatorname{rand}(N)$ gives the control to one of the activities in $N$ (by assigning randomly an element from $N$ to $\Pi$ ). The manager transits back to wait as soon as it has the control and $N$ is empty.

Since $\Theta(N)=\varnothing$, no activity would ever be executed. This is because fulfilling activities requests is the role of the control task that we do not represent here. Therefore, the manager performs the operation $\operatorname{rrand}(N)$ to initialize $N$ randomly, over the set of activities $T$ is in charge of; while respecting the condition $(A \in N \wedge B \in N) \Rightarrow(A \neq$ 
$B)$. The operation $\operatorname{rrand}(N)$ covers all the possible evolutions of tasks, as the resulting set of configurations of $N$ is a superset of that obtained when a control task is present (details in [11]). Note how the guard on the edge from wait to manage does not contain

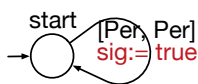

Fig. 4: Timer TTD

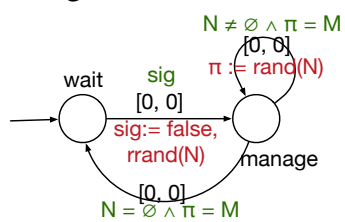

Fig. 5: Manager TTD
A

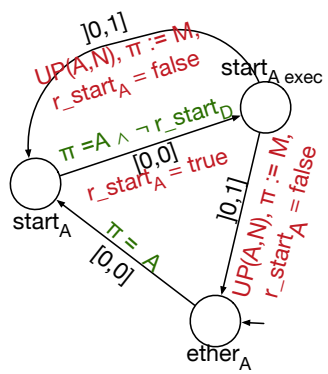

B

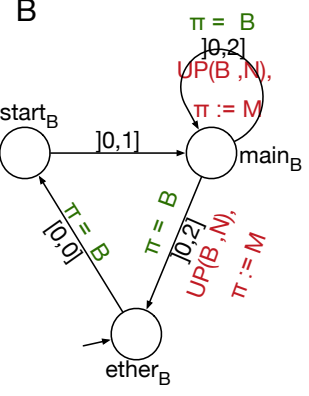

Fig. 6: Activities A and B in task T

the clause $\Pi=M$ because this is always true at vertex wait $(\Theta(\Pi)=M$ and the manager cannot lose the control at vertex wait).

Definition 3 Activities semantics. The operational semantics of an activity $\left\langle C_{A}, W_{A}, T_{A}, T_{A}^{P}\right\rangle$ (Sect. 3.2.1) is given by a TTD such that:

- Vertices $V:$ each $c \in C_{A}$ is mapped to a vertex $c \in V$. A vertex $c_{\text {exec }} \in V$ is added for each thread-unsafe codel $c\left(\mu(c) \neq \varnothing\right.$, Sect. 3.2.3). The initial vertex $v_{0}$ is ether ${ }_{A}$,

- Edges $E=E^{N} \cup E^{A}$ are nominal (in $E^{N}$ ) or additional (in $E^{A}$ ):

- $E^{N}$ : each transition $c \rightarrow c^{\prime}$ in $T_{A}$ is mapped to an edge $c \rightarrow c^{\prime}$ (resp. $c_{\text {exec }} \rightarrow c^{\prime}$ ) in $E^{N}$ if $\mu(c)=\varnothing$ (resp. otherwise). We distinguish three disjoint sets of nominal edges: $E^{N}=E^{P} \cup E^{T} \cup E^{X} . E^{P}$ is the set of pause edges that maps the set of pause transitions $T^{P} ; E^{T}$ is the set of termination edges of the form $\rightarrow$ ether and $E^{X}$ the set of the remaining (execution) edges.

- $E^{A}=E^{S} \cup E^{M}$ where $E^{S}$ contains the starting edge ether $\rightarrow$ start and $E^{M}$ the mutual exclusion edges of the form $c \rightarrow c_{\text {exec }}$ (for each thread-unsafe codel $c$ ).

- Time intervals $\left.I: I(e)=] 0, W_{A}(c)\right]$ iff $e \in E^{N}(I(e)=[0,0]$ otherwise $)$.

Now we define the guards and operations:

- Each edge in $E^{T} \cup E^{P}$ is augmented with the operation $\Pi:=M$ and the operation $U P(A, N)$ that removes $A$ (the activity "name") from $N$,

- The edge in $E^{S}$, and each edge $c \rightarrow$ in $E^{N} \cup E^{M}$ such that exists an edge $\rightarrow c$ in $E^{P}$, are guarded with $\Pi=A$,

- Each edge $c \rightarrow$ in $E^{M}$ is augmented with the operation $r_{-} c:=$ true (see shared variables in Sect. 3.3.1).

Finally, (i) the guard of each edge $c \rightarrow$ in $E^{M}$ is conjuncted with the expression $\forall c^{\prime} \in \mu(c): \neg r_{-} c^{\prime}$ and (ii) $r_{-} c:=$ false is added to the operations of each edge $c_{\text {exec }} \rightarrow$ in $E^{N}$.

Nominal edges map transitions that the programmer specifies, while additional edges reflect actions enforced by $\mathrm{G}^{\mathrm{e}}{ }_{\mathrm{O}} \mathrm{M} 3$ to handle starting and concurrency. Edges are uniquely defined through their source and target vertices. For activities, this can be con- 
cluded from syntax, restrictions and semantics (Sect. 3.2.1, Sect. 3.2.4 and Definition 3). For the manager and the timer, it is shown in Fig. 4 and Fig. 5.

Let us illustrate through an example how activities evolve following these semantics, and how this coincides with the behavior in Sect. 2.1.1. We consider a component with two tasks $T$ and $T^{\prime} . T$ is in charge of two activities $A$ and $B$ (on which we focus) while $T^{\prime}$ is in charge of one activity $D$. We give the syntactical definitions of $A$ and $B$ :

Activity $A$

- $C_{A}=\left\{\right.$ start $_{A}$, ether $\left._{A}\right\}$,

- $W_{A}\left(\right.$ start $\left._{A}\right)=1$,

- $T_{A}=\left\{\operatorname{start}_{A} \rightarrow \operatorname{start}_{A}\right.$, start $_{A} \rightarrow$ ether $\left._{A}\right\}$,

- $T_{A}^{P}=\left\{\right.$ start $\left._{A} \rightarrow \operatorname{start}_{A}\right\}$.
Activity $B$

- $C_{B}=\left\{\right.$ start $_{B}$, main $_{B}$, ether $\left._{B}\right\}$,

- $W_{B}\left(\operatorname{start}_{B}\right)=1, W_{B}\left(\operatorname{main}_{B}\right)=2$,

- $T_{B}=\left\{\operatorname{start}_{B} \rightarrow \operatorname{main}_{B}\right.$, main $_{B} \rightarrow$ main $_{B}$, main $_{B} \rightarrow$ ether $\left._{B}\right\}$, - $T_{B}^{P}=\left\{\operatorname{main}_{B} \rightarrow \operatorname{main}_{B}\right\}$.

Now, because of the mutual exclusion between $T$ and $T^{\prime}$, the start codels of $A$ (in $T$ ) and $D$ (in $T^{\prime}$ ) are in conflict: $\mu\left(\right.$ start $\left._{A}\right)=\left\{\right.$ start $\left._{D}\right\}$ (and symmetrically $\mu\left(\right.$ start $\left._{D}\right)=\left\{\right.$ start $\left.\left._{A}\right\}\right)$. The remaining codels are thread safe.

We apply Definition 3 to get the TTDs of $A$ and $B$ in Fig. 6 evolving within $T$ (the manager and timer (generic) TTDs are given in Fig. 5 and Fig. 4, respectively). Starting an activity, from ether or wherever it was paused last, is subject to having the control through $\Pi$ (e.g. edge ether $_{B} \rightarrow$ start $\left._{B}\right)$. At the end of execution, either by pausing (e.g. edge $\operatorname{main}_{B} \rightarrow$ main $_{B}$ ) or terminating (e.g. edge start $_{A \text { exec }} \rightarrow$ ether $A$ ), the control is given back to the manager $(\Pi:=M)$, and the activity removes its "name" from $N$ $(U P()$, no further execution for this activity in this cycle). $\Pi$ ensures thus a sequential behavior within the same task, that is between the manager and each $A$ in $\mathcal{A}$ (no two edges in two different TTDs can be enabled simultaneously).

At the codels level, outgoing edges of vertices $c$ (the underlying codel is thread safe, e.g. start $_{B}$ ) and $c_{\text {exec }}$ (otherwise, e.g. start $A$ ) are associated with the interval ] $0, W(c)]$ to reflect that the execution of a codel takes between a non-null time and its WCET. Boolean expressions involving $r_{-} c^{\prime}$ variables, which take part in the guards on edges $c \rightarrow c_{\text {exec }}$, prevent the thread-unsafe codel $c$ to execute if there is at least a codel in $\mu(c)$ that is already running, and the time interval $[0,0]$ allows it to execute as soon as this is no longer the case. For instance, the guard on $\operatorname{start}_{A} \rightarrow \operatorname{start}_{A}$ exec disables this very edge (even when $A$ has the control) as long as the activity $D$ (in the concurrent task $T^{\prime}$, not shown here) is at vertex $\operatorname{start}_{D}$ exec (denoting the execution of $\operatorname{start}_{D}$ ), captured through the truth of the Boolean $r_{-}$start $_{D}$. Similarly, operations $r_{-} c:=$ true on edges $c \rightarrow c_{\text {exec }}$ prevent thread-unsafe codels in $\mu(c)$ to run in parallel with $c$ (e.g. $r_{-}$start $_{A}:=$ true on start $_{A} \rightarrow$ start $_{A}$ exec $)$. Finally, operation $r_{-} c:=$ false on edges of the form $c_{\text {exec }} \rightarrow\left(\right.$ e.g. $r_{-}$start $t_{A}:=$ false $)$ allow activities with codels in conflict with $c$ to capture the end of execution of $c$ through the falseness of $r_{-} c$.

\section{Translation}

TTS semantics are translated to DUTA in order to automatically map Gen ${ }^{\mathrm{O}} \mathrm{M}$ to UPPAAL and UPPAAL-SMC. We show the translation for activities, since it is rather straightforward for the manager and the timer (Fig. 7).

Mapping intervals into clock constraints and \edges may lead to incorrect translations, as shown in Fig. 8 (activity B). Indeed, if $B_{t a}$ is paused (taking main $_{B} \rightarrow \operatorname{main}_{B}$ ), 


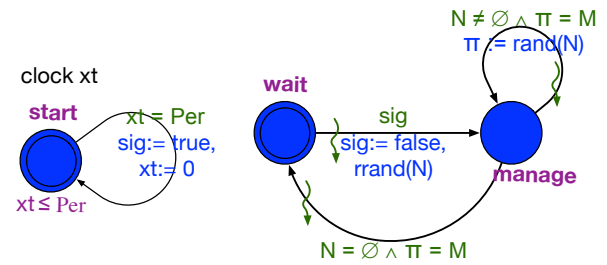

Fig. 7: DUTA translation of manager and timer

it will timelock after 2 time units unless it resumes the control before then (all outgoing edges from location main $_{B}$ are disabled). This is encountered when there is a vertex in the TTD that (i) maps a thread-safe codel and (ii) is the target of a pause edge. This problem is due to clocks evolving independently from edges enabledness in DUTA (in contrast to intervals in TTDs). We propose a generic translation for all activities.

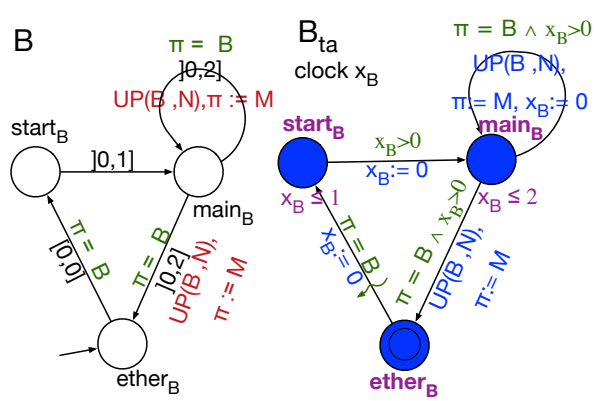

Fig. 8: Incorrect translation (activity $B$ )

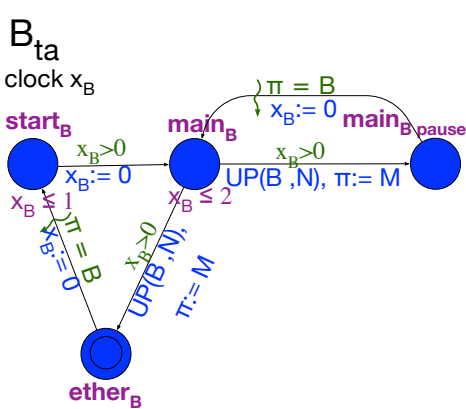

Fig. 9: Correct translation (activity $B$ )

Definition 4 Activities $A_{t a}$ (DUTA). The DUTA translation $A_{t a}$ of the TTD A (Definition 3), is given by the following rules:

- Clocks: $A_{t a}$ has a clock $x_{A}$, whose initial valuation is zero,

- Locations: Each vertex $c$ in $A$ of a thread-safe codel c s.t. there exists $\rightarrow c$ in $T^{P}$ is mapped to two locations $c$ and $c_{\text {pause. }}$ Each remaining vertex in $A$ is mapped to a location with the same name. Each location $c$ that maps a vertex $c \neq$ ether of a thread-safe codel is associated with an invariant $x_{A} \leq \uparrow I(c \rightarrow)$ with $c \rightarrow$ any outgoing edge of $c$. The same invariant rule is applied to each location $c_{e x e c}$,

- Edges: - Each pause edge $c \stackrel{g, o p}{\longrightarrow} c^{\prime}$ in $A$ s.t. $c^{\prime}$ is thread safe is mapped to an edge $c \stackrel{x_{A}>0, o p}{\longrightarrow} c_{\text {pause }}^{\prime}$, and an eager edge $c_{\text {pause }}^{\prime} \stackrel{g, x:=0}{\longrightarrow} c^{\prime}$ is added.

- Each remaining edge in $A$ is mapped to an edge in $A_{t a}$ with the same source and target, where: (1) intervals $[0,0]$ are mapped into $\{$ edges, (2) each outgoing (resp. incoming) edge of a location associated with an invariant is guarded (resp. augmented) with $x_{A}>0$ (resp. with $x_{A}:=0$ ), then (3) guards (resp. operations) associated with each edge result from the conjunction (resp. sequencing) of guards (resp. operations) of its TTD counterpart and the guards (resp. resets) over clocks.

These rules allow clocks to evolve unboundedly at locations $c_{\text {pause }}$ (when the activity is paused). Resuming the activity is then equivalent to taking the edge $c_{\text {pause }} \rightarrow c$ with a clock reset to count the WCET of $c$ starting from 0 , which we may see when applying Definition 4 to activity $B$ (Fig. 9). 
Translation soundness: DUTA models must be faithful to the $\mathrm{G}^{\mathrm{en}}{ }_{\mathrm{oM}} \mathrm{M}$ semantics. We use weak timed bisimulation to prove that the translation is sound. Details on the proof may be found in [11].

\section{Automatic mapping}

We see how the DUTA models are automatically mapped into UPPAAL and UPPAALSMC. In order to do so, we first present the current implementation.

Implementation: In the actual implementation (either in ROS-Comm or PocoLibs middleware), the set of activities to execute $(N)$ is substituted with an array run of size $n=|\mathcal{A}|$ (the number of activities in the task) of records, starting at index 0. Each record is composed of two fields: an activity "name" $m$ and its "status" $s$, that may be requested ( $r$ ) or idle ( $d$ ), equivalent, respectively, to $A \in N$ and $A \notin N$ in the semantics. The operation $\operatorname{arand}(t)$ initializes the status $s$ fields of array $t$ randomly. The variable $i$, initially equal to 0 , ranges from 0 to $n$. The function $n \operatorname{ext}(t, b)$ browses the array $t$, starting from index $b$, and returns the index of the first element with $s=r(|t|$ if such an element is not found or $b=|t|$ ).

The implementation of a task is then derived from its semantics as follows. For any activity $A$, each operation $U P(A, N)$ is replaced by $i:=i+1, i:=n e x t(r u n, i)$. In the manager, the guard $N \neq \varnothing$ (resp. $N=\varnothing$ ) is replaced by $i \neq n$ (resp. $i=n$ ), the operation $\Pi:=\operatorname{rand}(N)$ by $\Pi:=\operatorname{run}[i] . m$, and the operation $\operatorname{rrand}(N)$ by $\operatorname{arand}($ run $), i:=n \operatorname{ext}($ run,$i)$ (in reality, the run array is updated by the control task, not considered in our presentation). Finally, the edge manage $\rightarrow$ wait in the manager is augmented with the operation $i:=0$. Accordingly, the implementation model of task $T$ (Fig. 5, 4, 6) is given in Fig. 10. Trivially, the semantics (allowing random "scheduling" of activities) is a superset of the implementation (where the order of execution of activities is predefined when initializing names fields $(m)$ in run). The random scheduling at the semantics level allows to derive different implementations if needed. For DUTA, it is sufficient to apply the TTD-DUTA translation rules. Fig. 11 gives the DUTA implementation of activity $A$ (Fig. 10).
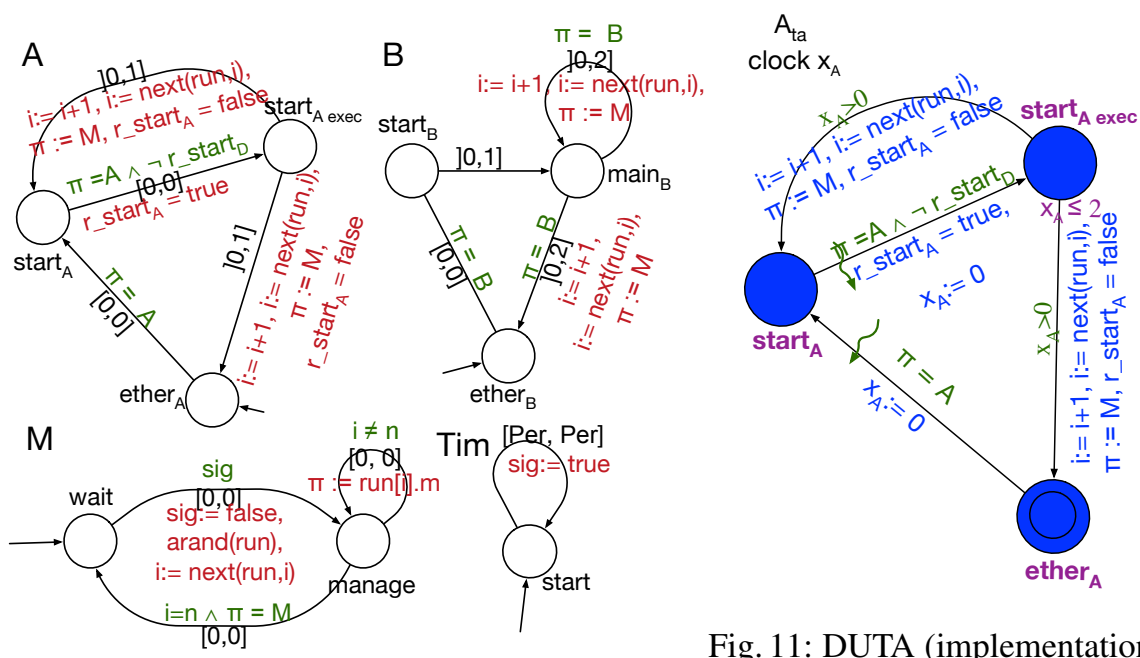

Fig. 10: TTDs in task $T$ (implementation).

Fig. 11: DUTA (implementation) of activity $A$. 


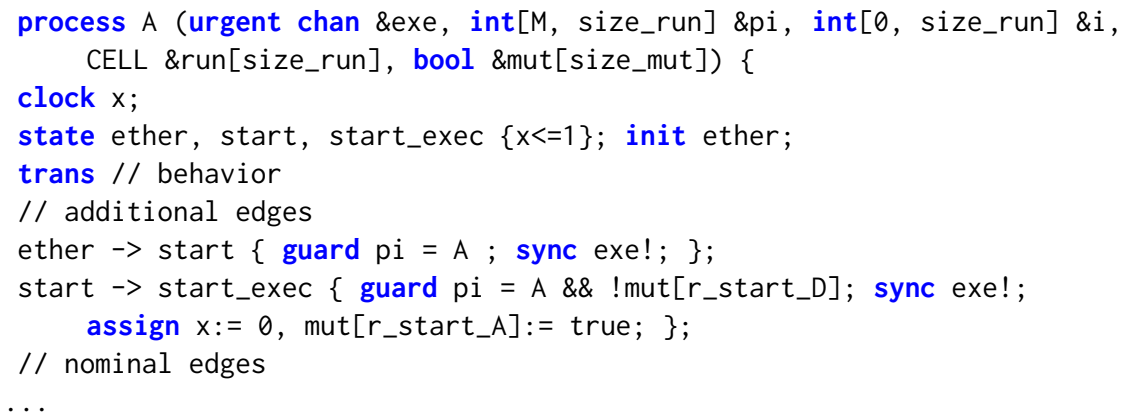

Listing 3: Process $A$ (UPPAAL)

\subsection{Mapping to UPPAAL}

We see how to model an activity. First, we deal with urgent edges (UPPAAL only allows urgent channels, Sect. 2.2). We add a process urgency and synchronize its unique edge, over an urgent channel exe, with each eager edge in the activity (and with all eager edges in the system):

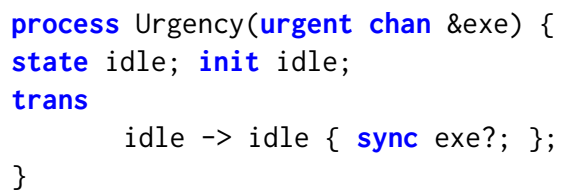

Now we can model $e . g$. activity $A$. Listing. 3 is a partial UPPAAL model of $A$ (only additional edges are shown). Constant $M=0$ denotes the manager, so $\Pi$ ranges over $[M$, size_run] (line 1$)$ where activity names are encoded in turn as constant integers in this range. CELL is the record type for run (line 1) and mut is an array that facilitates implementing mutual exclusion variables ( $r_{-} c$ becomes $m u t\left[r_{-} c\right]$, line 1,7).

\subsection{Automatic synthesis}

We generalize the approach for automatic synthesis using the template mechanism (Sect. 2.1.2). We develop a template that generates automatically the UPPAAL model for any Gen ${ }_{0} \mathrm{M} 3$ specification (made of any number of components). We show an example on how additional edges are generated for a given activity a (listing 4). The interpreter outputs everything as is, except what is enclosed in $<$ ' ' $>$ that it evaluates in Tcl, and in $<" ~ ">$ that it evaluates and outputs the result.

In lines 3 to 7, we check each outgoing transitions of each codel (keyword yields), and append the successor to the list $p$ if such transition is a pause. We also append the codel $\mathrm{C}$ to the list tu if its field mutex, which contains the codels $\mathrm{C}$ is in conflict with, is not empty. Therefore, $p$ contains all the codels targeted by a pause and tu all threadunsafe codels in a. At line 11, we generate the starting edge, then the mutual exclusion edges from line 12 to 20, where, for each thread-unsafe codel c, we add the guard on having the control through $\Pi$ if $c$ is also in $p$ (applying Definition 4 and inductively Definition 3). The task name is added to distinguish variables in different tasks.

Extending to UPPAAL-SMC: Implementation templates (Sect. 2.1.2) generate, for each transition in each activity, a line with the number of its occurrences: 


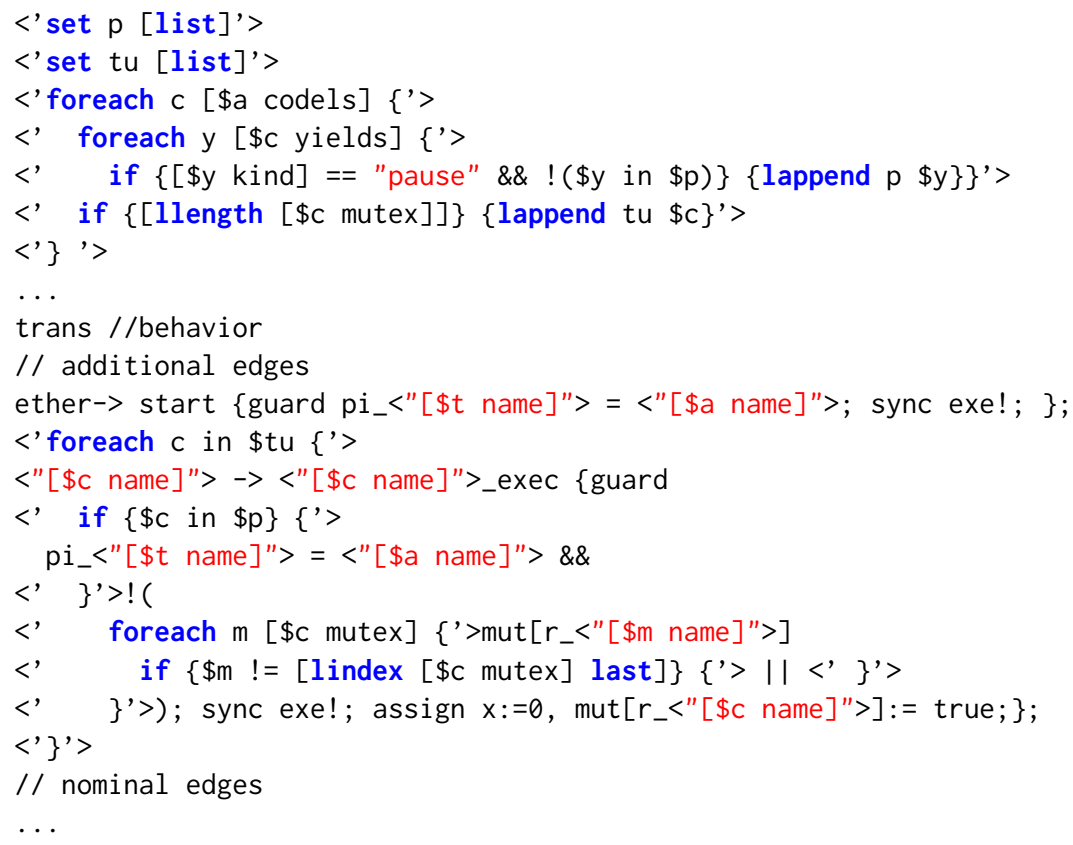

Listing 4: Generating additional edges (for an activity $a$ in task $t$ )

A .proba file is thus constructed, then passed as an argument to the UPPAAL-SMC template, together with the $\mathrm{G}^{\mathrm{en}} \mathrm{M} 3$ specification. Listing 5 shows an excerpt of the UPPAAL-SMC template . For simplicity, we only show the case where the source codel is thread safe and none of its outgoing transitions is pause or termination. Line 3 conditions adding probabilities by the existence of more than one successor. Line 5 connects the edge to a branchpoint (as shown in Sect. 5.1). Lines 6-8 generate the outgoing edges of the branchpoint and extract occurrences from the .proba file.

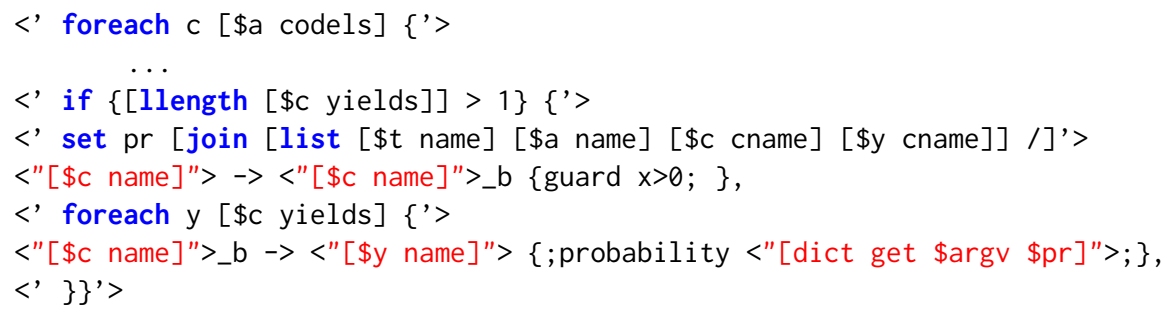

Listing 5: Generating probabilistic transitions (for an activity $a$ in task $t$ )

\section{Verification results}

We use the automatically generated models (Sect. 5) to specify and verify important real-time properties on the quadcopter case study (Sect. 2.1.3). Experiments are carried out on a laptop (Intel Core i7; 16 GB of RAM). Tasks are assigned to independent cores on the hardware. Experiments, with instructions on how to reproduce them, are freely clonable from https://github.com/Mo-F/uppaal-smc-exp. 


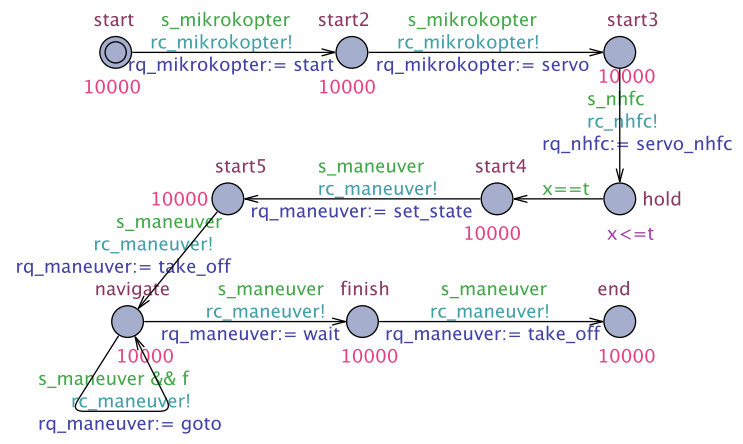

Fig. 12: UPPAAL-SMC client (quadcopter navigation).

\subsection{Model checking}

With UPPAAL, we get the same results as with the Fiacre template in [8]: the stationary flight application (excluding the component MANEUVER) scales, while the navigation application (involving all components) does not. We use UPPAAL-SMC for the latter.

\subsection{Statistical model checking}

As seen in Sect. 2, components need to receive requests from clients to run. For that, we add a client to ensure a navigation application (see below). The automatically generated UPPAAL-SMC model of the quadcopter plus the added client make 36 complex processes overall, on which we carry out the statistical verification.

6.2.1 Client The client (Fig. 12) uses urgent channels $r c_{-} X$ ( $X$ is a component) to send activities requests to components, through $r q_{-} X$ variables. Since UPPAAL-SMC supports only broadcast channels, we guard each channel $r c_{-} X$ with the Boolean $s_{-} X$, true only when $X$ is ready to receive a request (which forces a rendezvous behavior). Location hold is for waiting an amount $t$ between sending servoing requests (NHFC and MIKROKOPTER) and taking off (MANEUVER), as servoing must have already started before taking off (which is an important property to verify). Exponential rates are required on invariant-free locations (high rates imply a high probability to leave the location at smaller time values, but values are unimportant here because of the urgencies enforced by $r c_{-} X$ channels). The self-loop at location navigate enables, using the Boolean $f$, issuing a new goto request each time the last goto activity (to navigate) has ended (goal invalid, reached, or unreachable). From the same location, a request wait then take_off can be sent (to land). The client covers thus all the possible scenarios of navigation.

6.2.2 Properties of interest The following properties are crucial such that accidents may occur if they are not satisfied.

Readiness: When requests are sent to MANEUVER, the previously requested activities from MIKROKOPTER and NHFC must have already started executing. Find the minimum value of $t$ to satisfy this property with the highest possible probability.

Schedulability: Estimate the probability of schedulability of periodic tasks in the critical components POM, MIKROKOPTER and NHFC.

6.2.3 Verification with UPPAAL-SMC Statistical parameters are set to a high confidence $(0.98)$ and precision $(0.005)$, and the runs are bounded to $b=10 \mathrm{~s}$. 
Readiness: Readiness is typically a bounded response property, not supported by UPPAAL-SMC. We propose an alternative using the Until operator. An activity starts once its codel start begins executing, which is equivalent to reaching the location start_exec (since none of the codels start in this context is thread safe). Therefore, the client "cl" must not reach location start4 (from which it sends requests to MANEUVER) before locations start_exec of each previously requested activity (start and servo (MIKROKOPTER) and servo in NHFC) is reached. Readiness boils down then to the conjunction of the three Until properties in listing 6.

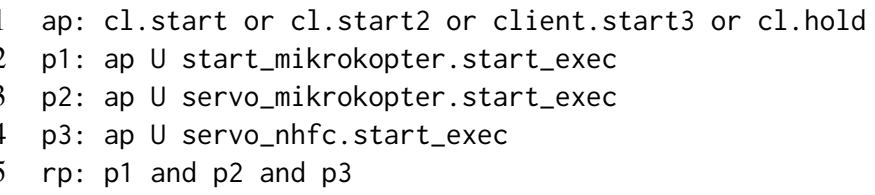

Listing 6: Readiness property $r p$

Note that attempting to reduce these properties to only one using the conjunction of their right terms would result in a stricter property (e.g. start_exec of servo may be left before start_exec of servo_nhfc is reached). We tune $t$ starting from $1 \mathrm{~ms}$. The highest possible probability is returned by the verifier ( $\geq 99 \%$ considering the precision, $0.005 \pm 0.005$ ) for all of the three properties as soon as $t$ is equal to $8 \mathrm{~ms}$. Results for $p 3$ for different values of $t$ are given in table 1 . Therefore, in order to ensure a high probability of satisfying Readiness, $t$ may have any value larger than $8 \mathrm{~ms}$. We fix it to $1 \mathrm{~s}$.

\begin{tabular}{|c|c|c|c|}
\hline $\mathrm{t}(\mathrm{ms})$ & Results & Runs & Time \\
\hline 7 & $\operatorname{Pr} \in[0.98,0.99]$ & 3279 & 12 \\
\hline 8 & $\operatorname{Pr} \in[0.99,1]$ & 1595 & 6 \\
\hline 100 & $\operatorname{Pr} \in[0.99,1]$ & 390 & 3 \\
\hline
\end{tabular}

Table 1: Analysis results for $\mathrm{p} 3$ (li-

sting 6) with the query $\operatorname{Pr}[<=b] p 3$.

\begin{tabular}{|c|c|c|c|c|}
\hline Task & Query & Results & Runs & Time \\
\hline io & $\operatorname{Pr}[<=b] v s_{i o}$ & $\operatorname{Pr} \in[0,0.01]$ & 390 & 966 \\
\hline filter & $\operatorname{Pr}[<=b] v s_{\text {filter }}$ & $\operatorname{Pr} \in[0,0.01]$ & 390 & 962 \\
\hline
\end{tabular}

Table 2: Analysis results for schedulability (POM)

Schedulability: It is reduced to a reachability property. Indeed, it is sufficient to verify that whenever the manager is executing activities (at location manage), no new period signal is received ( $\mathrm{sig}$ is false), see Fig. 7. The probability of violating this property is the lowest possible for all tasks of the critical components POM, MIKROKOPTER and $\operatorname{NHFC}(\leq 1 \%)$. Examples of results on POM tasks are given in table 2 with $v s_{T}$ being the violation of schedulability of task $T:<>$ manager_T.manage and sig_T.

6.2.4 Discussion While we cannot verify some properties in a precise way (due to scalability issues with model checking), the results we get with UPPAAL-SMC are encouraging. We verify important properties up to a high probability, which is better than classical scenario-based testing. The verification is cost effective: around 15 minutes in the worst case, and a remarkably low memory consumption (less than $15 \mathrm{mb}$ ). Nevertheless, two main issues are encountered, besides non exhaustivity. First, though 99\% is fair for this application, we generally lack precise requirements expressed probabilistically in the robotics domain. Second, the expressiveness of UPPAAL-SMC query language is limited (e.g. bounded response properties are not supported). While we often manage, with some artefacts, to verify closer alternatives, such artefacts need a proficiency with formal languages that robotic practitioners do not possess. 


\section{Related work}

Model checking: The synchronous language ESTEREL [3] is used in some modelchecking-based verification works such as [18,30,31], where the robotic specifications are either translated by hand to, or hard-coded in ESTEREL. Efforts such as [24] rely on automatic translation of RoboChart models into CSP [27] in order to verify realtime properties. However, RoboChart is not a robotic framework (its models are not executable on robotic platforms). That is, robotic applications, initially specified in a robotic framework, need to be modeled first in RoboChart, then translated into CSP. An attempt to formalize ROS components is developed in [13] where UPPAAL is used to verify buffer-related properties (no overflow). Only the message passing part (publisher/subscriber) is modeled, manually, and crucial bounded response properties (e.g. messages are delivered within a bounded amount of time), are not verified. Our work distinguishes itself across three main aspects: (i) this is the first work that fully formalizes a robotic framework for functional-layer specifications, (ii) modeling is fully automatized and (iii) only real-world applications are analyzed.

Statistical \& probabilistic model checking: Real-time statistical/probabilistic model checking has been used to verify systems in various domains such as communication protocols [21], railway systems [6] and decisional robotics [29]. At the functional layer of robotic systems, statistical and probabilistic model checkers are seldom used. The work presented in [14] is a notable exception. ROS graphs are formalized in an ad-hoc fashion (no operational semantics given), then, on an autonomous vehicle case study, PRISM [20] estimates the probability of finding an object in a bounded amount of time. To the best of our knowledge, our work presented here is the first that applies realtime statistical model checking to complex, concurrent functional layer, where formal models are sound and automatic. The choice of UPPAAL-SMC is motivated by the fact that the automatic translation gives us the opportunity to use regular UPPAAL and resort to UPPAAL-SMC when models do not scale.

Comparison to our previous work: In our previous efforts to verify the quadcopter, model checking scaled only for the stationary flight, excluding the MANEUVER component $[8,9]$. This is the first work that verifies the navigation application, involving all the components, through sound and automatic bridging with UPPAAL-SMC.

\section{Conclusion}

We propose in this paper automatic and sound generation of formal models from robotic specifications, and obtain encouraging results on a real application. Our contributions advance the state of the art toward a correct and practical verification of robotic systems.

However, it is difficult to set the probabilities for properties because we lack this kind of requirements in robotics. We need to investigate further this problem. Moreover, the restricted query language of UPPAAL-SMC forced us to reason on alternatives using the supported operators only. For a robotic programmer, this could be discouraging since it requires a good knowledge of the tool, the query language and the underlying logic. A possible future work consists therefore in developing query-to-query transformations that are transparent to the practitioner. Finally, we are interested in verifying some hardware-related properties using SMC such as energy consumption (as in [28]). 


\section{References}

1. The PocoLibs middleware https://git.openrobots.org/projects/pocolibs.

2. G. Behrmann, A. David, and K.G. Larsen. A tutorial on UPPAAL. In Formal Methods for the Design of Real-Time Systems, pages 200-236. 2004.

3. G. Berry. The Esterel v5 language primer: version v5_91. Centre de mathématiques appliquées, Ecole des mines and INRIA, 2000.

4. S. Bornot, J. Sifakis, and S. Tripakis. Modeling urgency in timed systems. In International Symposium on Compositionality (ISC): the significant difference, pages 103-129. 1998.

5. P. Bulychev, A. David, K-G. Larsen, A. Legay, G. Li, D. B. Poulsen, and A. Stainer. Monitorbased statistical model checking for weighted metric temporal logic. In International Conference on Logic for Programming Artificial Intelligence and Reasoning (LPAR), pages 168182. Springer, 2012.

6. Q. Cappart, C. Limbrée, P. Schaus, J. Quilbeuf, L-M. Traonouez, and Axel Legay. Verification of interlocking systems using statistical model checking. In 2017 IEEE 18th International Symposium on High Assurance Systems Engineering (HASE), pages 61-68. IEEE, 2017.

7. A. Cimatti, M. Roveri, and P. Bertoli. Conformant planning via symbolic model checking and heuristic search. Artificial Intelligence, 159(1-2):127-206, 2004.

8. M. Foughali. Toward a Correct-and-Scalable Verification of Concurrent Robotic Systems: Insights on Formalisms and Tools. In International Conference on Application of Concurrency to System Design (ACSD), pages 29-38, 2017.

9. M. Foughali, B. Berthomieu, S. Dal Zilio, P.-E. Hladik, F. Ingrand, and A. Mallet. Formal verification of complex robotic systems on resource-constrained platforms. In International Conference on Formal Methods in Software Engineering (FormaliSE), pages 2-9, 2018.

10. M. Foughali, B. Berthomieu, S. Dal Zilio, F. Ingrand, and A. Mallet. Model checking realtime properties on the functional layer of autonomous robots. In International Conference on Formal Engineering Methods (ICFEM), pages 383-399. Springer, 2016.

11. M. Foughali, S. Dal Zilio, and F. Ingrand. On the Semantics of the GenoM3 Framework. Technical report, LAAS-CNRS, 2019.

12. D. Hähnel, W. Burgard, and G. Lakemeyer. GOLEX - bridging the gap between logic (GOLOG) and a real robot. In Annual Conference on Artificial Intelligence, pages 165-176. Springer, 1998.

13. R. Halder, J. Proença, N. Macedo, and A. Santos. Formal verification of ROS-based robotic applications using timed-automata. In International Conference on Formal Methods in Software Engineering (FormaliSE), pages 44-50. IEEE/ACM, 2017.

14. M. Hazim, H. Qu, and S. Veres. Testing, verification and improvements of timeliness in ROS processes. In Annual Conference Towards Autonomous Robotic Systems (TAROS), pages 146-157, 2016.

15. T. Henzinger, Z. Manna, and A. Pnueli. Timed transition systems. In Research and Education in Concurrent Systems, pages 226-251, 1991.

16. T. Henzinger, X. Nicollin, J. Sifakis, and S. Yovine. Symbolic model checking for real-time systems. Information and computation, 111(2):193-244, 1994.

17. F. Ingrand and M. Ghallab. Deliberation for autonomous robots: A survey. Artificial Intelligence, 247:10-44, 2017.

18. M. Kim and K. Kang. Formal construction and verification of home service robots: A case study. In International Symposium on Automated Technology for Verification and Analysis (ATVA), pages 429-443. Springer, 2005.

19. H. Kress-Gazit, T. Wongpiromsarn, and U. Topcu. Correct, reactive, high-level robot control. IEEE Robotics \& Automation Magazine, 18(3):65-74, 2011. 
20. M. Kwiatkowska, G. Norman, and D. Parker. PRISM 4.0: Verification of probabilistic realtime systems. In International Conference on Computer-Aided Verification (CAV), pages 585-591. Springer, 2011.

21. M. Kwiatkowska, G. Norman, and J. Sproston. Probabilistic model checking of deadline properties in the IEEE 1394 FireWire root contention protocol. Formal Aspects of Computing, 14:295-318, 2003.

22. A. Legay, B. Delahaye, and S. Bensalem. Statistical model checking: An overview. In International Conference on Runtime Verification (RV), pages 122-135. Springer, 2010.

23. A. Mallet, C. Pasteur, M. Herrb, S. Lemaignan, and F. Ingrand. GenoM3: Building middleware-independent robotic components. In International Conference on Robotics and Automation (ICRA), pages 4627-4632. IEEE, 2010.

24. A. Miyazawa, P. Ribeiro, W. Li, A. Cavalcanti, and J. Timmis. Automatic property checking of robotic applications. In International Conference on Intelligent Robots and Systems (IROS), pages 3869-3876. IEEE, 2017.

25. C. Pecheur. Verification and validation of autonomy software at NASA. Technical report, NASA Ames Research Center, 2000.

26. M. Quigley, B. Gerkey, K. Conley, J. Faust, T. Foote, J. Leibs, E. Berger, R. Wheeler, and A. Ng. ROS: an open-source Robot Operating System. In ICRA workshop on open source software, page 5, 2009.

27. A. Roscoe. Understanding concurrent systems. Springer Science \& Business Media, 2010.

28. C. Seceleanu, A. Vulgarakis, and P. Pettersson. REMES: A resource model for embedded systems. In International Conference on Engineering of Complex Computer Systems (ICECCS), pages 84-94, 2009.

29. T. Sekizawa, F. Otsuki, K. Ito, and K. Okano. Behavior verification of autonomous robot vehicle in consideration of errors and sisturbances. In International Computer Software and Applications Conference (COMPSAC), pages 550-555, 2015.

30. D. Simon, R. Pissard-Gibollet, and S. Arias. Orccad, a framework for safe robot control design and implementation. In National workshop on control architectures of robots: software approaches and issues (CAR), 2006.

31. A. Sowmya, D. Tsz-Wang So, and W. Hung Tang. Design of a mobile robot controller using Esterel tools. Electronic Notes in Theoretical Computer Science, 65(5):3-10, 2002.

32. N. Tomatis, G. Terrien, R. Piguet, D. Burnier, S. Bouabdallah, K. Arras, and R. Siegwart. Designing a secure and robust mobile interacting robot for the long term. In International Conference on Robotics and Automation (ICRA), pages 4246-4251. IEEE, 2003.

33. R. Volpe, I. Nesnas, T. Estlin, D. Mutz, R. Petras, and H. Das. The CLARAty architecture for robotic autonomy. In Aerospace Conference, pages 1-121, 2001. 